Trauma Berufskrankh 2013 · 15[Suppl 1]:75-80 DOI 10.1007/s10039-013-1938-5

(c) Springer-Verlag Berlin Heidelberg 2013
Online publiziert: 16. Mai 2013

\author{
A. Partenheimer ${ }^{1,2}$ \\ ${ }^{1}$ Klinik für Unfall- und Wiederherstellungschirurgie, Abteilung des BG-Unfallkrankenhauses Hamburg, \\ Diakoniekrankenhaus Friederikenstift gGmbH, Hannover \\ ${ }^{2}$ Mannschaftsarzt Hannover $96-1$. Fußballbundesliga, Hannover
}

\section{Konservative Behandlung von Muskelverletzungen}

\section{Rehabilitation und Grenzen}

\section{Grundlagen}

Der menschliche Körper besitzt mehr als 640 Skelettmuskeln. Sie bilden den größten Anteil des aktiven Bewegungsapparats und machen etwa 40-65\% des gesamten Körpergewichts aus.

Histologisch unterscheidet man die spontanen Muskeln, glatte Muskeln und die Herzmuskulatur, von der Skelettmuskulatur, welche eine sympathische Innervation zur Kontraktion benötigt.

Strukturell lässt sich der Muskel folgendermaßen beschreiben: Die Muskelfibrille setzt sich aus Sarkomeren zusammen. Eine Vielzahl an Muskelfibrillen wird durch das Endomysium internum zum Primärbündel zusammengefasst, von denen wiederum eine Vielzahl durch das Endomysium externum zum Sekundärbündel, der sog. Fleischfaser, gebündelt wird. Eine Vielzahl an von der Faszie umschlossenen Fleischfasern bildet dann den eigentlichen Muskel. Das Sarkomer ist der kontraktile Apparat der Muskulatur, in dem Aktin und Myosin, reguliert durch die Proteine Troponin und Tropomyosin, durch Bindung und Lösen der Proteine aneinander vorbeigleiten und dadurch das Sarkomer verkürzen, d. h. letztendlich den Muskel kontrahieren.

Funktionell-histologisch lässt sich die Muskulatur in die ST- („slow twitch“) oder auch Typ-1-Fasern, welche durch langsam zuckende/kontrahierende Dauerleistung mit begrenztem Kraftaufwand und aerobem Stoffwechsel charakterisiert sind, und die FT-Fasern („fast twitch“), die Typ-2-Fasern, welche schnell kontrahierend reagieren, unterscheiden. Letztere verbrauchen mehr Energie und ermüden schneller als die ST-Fasern. Die Typ-2-Fasern lassen sich weiter in Typ-2a-Fasern, welche ein ähnliches Verhalten wie Typ-1Fasern aufweisen, und die Typ-2b-Fasern, welche sehr kräftig sind und eine schnelle Kontraktilität besitzen, unterscheiden.

\section{Epidemiologie}

Im Fußball betreffen nahezu 92\% der Muskelverletzungen die untere Extremität. Hauptverletzungsregion hierbei ist die ischiokrurale Muskulatur, gefolgt von den Quadrizepsverletzungen und den Läsionen im Bereich der Leiste und des Unterschenkels. Die meisten dieser Verletzungen treten während des Wettkampfs auf [3].

\section{Risikofaktoren}

Hauptrisikofaktor ist mangelhaftes Aufwärmen mit hieraus resultierender relativer Minderdurchblutung und damit fehlender Aktivitätsbereitschaft der Muskelund Gelenkrezeptoren. Als weitere Risiken sind ungenügendes Training, Muskelermüdung durch fehlende Erholungsphasen bzw. zu viele Trainingseinheiten zu nennen. Auch vorangegangene Muskeleinrisse mit Narbenbildung oder bereits bestehende Muskelverhärtungen erhöhen das Risiko für Muskelverletzungen.

\section{Ursachen/Entstehung}

In der Mehrzahl der Fälle handelt es sich um Muskelkontraktions- bzw. -überlastungsverletzungen. Dies bedeutet, dass der Muskel über das physiologische Maß hinaus gedehnt wird und hierdurch eine strukturelle Schädigung erfährt. Entsprechende Bewegungsmuster sind ein plötzliches Anhalten bzw. Abbremsen, rasche Beschleunigungen sowie BremsBeschleunigungs-Kombinationen. Hierdurch kommt es zu einer kurzzeitig überhöhten Muskelschnellkraft mit maximaler Kontraktion. Neben diesen Mechanismen können auch direkte Traumen im Sinne von Kontusionen Muskelverletzungen hervorrufen.

\section{Anamnese}

Eine exakte Befragung des Verletzten ist unverzichtbar:

- Wobei ereignete sich die Verletzung?

- Gab es einen Kontakt mit einem Gegenspieler oder trat die Verletzung ohne Fremdeinwirkung auf?

- Was für einen Charakter hatte der initiale Schmerz?

War der Schmerz eher spitz stechend wie ein Nadel- oder Messerstich, spricht dies für eine akute Muskelschädigung. Ein mehr dumpfer und krampfartiger Schmerz ist Hinweis auf eine neurogene Schädigung. Ein plötzlich auftretender Schmerz deutet eher auf eine akute Schä- 


\section{Tab. 1 PECH-Schema}

\begin{tabular}{|lll} 
P & Pause & Die verletzte Region sollte sofort ruhig gestellt werden. \\
\hline E & Eis & Unmittelbare Kühlung des verletzten Bereichs mit Eiswasser („hot ice“ für 20 min) \\
\hline C & $\begin{array}{l}\text { Kom- } \\
\text { pression }\end{array}$ & $\begin{array}{l}\text { Neben der sofortigen Kühlung ist das Anlegen eines festen elastischen Verbands } \\
\text { erforderlich, um die Entwicklung eines Hämatoms schnellstmöglich einzudämmen. }\end{array}$ \\
\hline H & $\begin{array}{l}\text { Hochla- } \\
\text { gerung }\end{array}$ & $\begin{array}{l}\text { Hierdurch werden die Blutzufuhr in das verletzte Areal verringert und extrazelluläre } \\
\text { Flüssigkeit verbessert abtransportiert. }\end{array}$ \\
\hline
\end{tabular}

digung hin als ein sich langsam steigernder Schmerz.

Wichtig ist auch, in welchem Bereich des Muskels der Athlet das Schmerzempfinden hat, d. h. ob der Schmerz nur einen definierten kleinen punktförmigen Bereich oder den gesamten Muskel bzw. einen größeren Bereich desselben betrifft.

Neben der genauen Erhebung der Entstehung der Verletzung, der Schmerzart und -lage ist auch die Abklärung eines veränderten Trainingsverhaltens, z. B. bezüglich Intensität, Pausen oder Inhalten, von Bedeutung.

Zusätzlich muss stets die Historie des Patienten mit beleuchtet werden:

- Hatte der Verletzte in den vorangegangenen Tagen Schmerzen oder Probleme im Bereich der Wirbelsäule oder des Beckens?

- Gab es Anhalt für einen generalisierten Infekt?

- Wie waren die unspezifischen und spezifischen Laborwerte?

Die Zusammenschau all dieser Parameter erlaubt dem Untersucher bereits eine erste Einschätzung über die Verletzungsschwere und die Zuordnung in eher funktionelle oder eher strukturelle Muskelschädigung.

\section{Symptome}

Sie reichen von sofortigen, heftigen bis stechenden Schmerzen bis hin zu dumpfen ziehenden, sich langsam steigernden Schmerzen. Diese nehmen häufig über Nacht zu, da das sich entwickelnde Hämatom auf die umliegenden Strukturen drückt.

Häufig ist bei ausbleibender Kühlung oder aber nach einem Tag eine Schwellung palpierbar. Es kommt zu einer Bewegungseinschränkung der betroffenen Region bis hin zur vollständigen Bewegungsunfähigkeit. Bei der Palpation ist der betroffene Bereich meist deutlich to- nisiert und, je nach Ausmaß der Verletzung, kann auch eine tastbare Kontinuitätsunterbrechung imponieren.

\section{Therapie}

Die initiale Therapie richtet sich nach dem PECH-Schema (• Tab. 1):

Generell kommen sowohl lokale als auch systemische Maßnahmen zur Anwendung. Lokal sollten nach der initial erfolgten Behandlung nach dem PECHSchema ein elastischer Kompressionsoder ein Entlastungstapeverband angelegt werden. Dieser sollte bis zum Folgetag belassen werden. Zusätzlich ist je nach Verletzungsausmaß auch die Injektionstherapie des betreffenden Muskels indiziert. Ziel dieser Maßnahme sind die Hemmung der inflammatorischen Reaktion und ggf. auch die Reduktion des entstandenen Hämatoms sowie die Detonisierung des verletzten Muskels.

Neben der lokalen Behandlung spielt auch die systemische Therapie in der Versorgung von Muskelverletzungen eine wichtige Rolle. Hierbei kann eine Vielzahl an oral zu verabreichenden Medikamenten zur Anwendung kommen.

\section{Medikamente}

\section{Enzyme}

Bromelain ist ein pflanzliches, fibrinolytisch wirksames Enzym, welches die Freisetzung von Interleukinen und Phagozytosemediatoren hemmt. Es wirkt gerinnungs- und entzündungshemmend und hilft beim Abbau von Fibrin [1, 7].

Aescin ist ein standardisierter Extrakt aus den Samen der Rosskastanie. Es verändert die Permeabilität der Gefäßwände und wirkt somit antiexsudativ und ödemprotektiv. Zusätzlich hat es einen Zellmembran stabilisierenden Effekt [11].

\section{Vitamine}

Vor allem die Vitamine A, C und $\mathrm{E}$ sind in der Frühphase der Regeneration und Reparation von Bedeutung. Zum einen dienen sie als Koenzyme, zum anderen als Fänger freier Radikale.

\section{Nichtsteroidale Antiphlogistika (NSAR)}

Sie spielen in der Therapie von Muskelverletzungen eine wichtige Rolle, zum einen durch Schmerzreduktion, zum anderen durch Hemmung der Ausschüttung von Gewebemediatoren in der Akutphase. Die Wirkweise ist auf die Hemmung der Cyclooxygenase und die dadurch verminderte Synthese von Prostaglandinen zurückzuführen.

\section{Physiotherapie}

In der Behandlung von Muskelverletzungen kommen, in Abhängigkeit vom Heilungsverlauf und vom Abstand zum Verletzungszeitpunkt, alle gängigen und üblichen Formen der Physiotherapie zum Einsatz, zu Beginn die physikalischen Therapien und Elektrotherapien, im weiteren Verlauf Strain-Counterstrain- sowie osteopathische Techniken, wie das Fasziendistorsionsmodell (FDM) nach Typaldos [10] im Bereich der angrenzenden Gelenke und Muskulatur.

Unterstützt wird die Physiotherapie durch Tapeverbände. Diese werden im initialen Stadium als stützende und Kompressionsverbände angelegt, im weiteren Verlauf kommen auch Kinesiotapeanlagen, sowohl als Lymph- als auch als Muskeltape, zur Unterstützung in behandlungsfreier Zeit zur Anwendung.

\section{Ziel}

Ziel aller Behandlungsmaßnahmen ist, die sekundäre Destruktionsphase soweit wie möglich einzudämmen und die sich anschließende Reparations- und Remodellierungsphase [4] zu verkürzen und zu unterstützen.

\section{Einteilung der Muskelverletzungen}

Hierfür stehen mehrere Klassifikationen zur Verfügung. 


\section{Betroffene Strukturen}

Eine Möglichkeit der Einteilung ist die Analyse der Lokalisation, also die Unterscheidung in Verletzungen, die den Muskelbauch, den Muskel-Sehnen-Übergang, die Sehne oder den Sehnen-KnochenÜbergang betreffen [3].

\section{Schwere der Verletzung}

Auch Klassifikationen in Bezug auf das Ausmaß der Verletzung sind gebräuchlich. Engelhardt et al. [5] veröffentlichten 2009 eine erste deutschsprachige Klassifikation dieser Art. Sie unterschieden 4 Grade, wobei Grad I dem Riss einzelner Muskelfasern von unter 5\% und Grad IV dem kompletten Riss der Muskulatur und Faszie mit vollständigem Funktionsverlust entsprechen.

\section{Ursache und Ausmaß der Verletzung}

Müller-Wohlfahrt et al. [8] entwickelten eine Klassifikation, die sowohl die Entstehung als auch das Ausmaß der Verletzung berücksichtigt und zudem einen direkten Behandlungsansatz liefert. Auch diese Klassifikation untergliedert sich aufsteigend nach der Verletzungsschwere.

Die leichteste Form der Muskelverletzung nach Müller-Wohlfart et al. [8] ist die Muskelverhärtung, anhand deren Ursache Ermüdung der Muskulatur an sich oder Verhärtung aufgrund neurogener Störungen unterschieden werden. Es folgen die Muskelzerrung, der Muskelfaserriss, der Muskelbündelriss und als schwerste Form der Verletzung der komplette Muskelriss bzw. der sehnige Muskelausriss.

Aufgrund der scharfen Trennung der Verletzungsschwere und der sich daraus ergebenden Konsequenzen in der Behandlung kommt diese Klassifikation bevorzugt zu Anwendung.

\section{Typ la: Muskelverhärtung durch Ermüdung}

Es kommt zu einer Verhärtung durch Überlastung und Ermüdung des Muskels. Die Beschwerden des Sportlers äußern sich in Form von ziehenden bis hin $\mathrm{zu}$ stechenden Schmerzen und einem zunehmenden Spannungsgefühl. In der Thera-

Trauma Berufskrankh 2013 · 15[Suppl 1]:75-80 DOI 10.1007/s10039-013-1938-5

(c) Springer-Verlag Berlin Heidelberg 2013

\section{A. Partenheimer \\ Konservative Behandlung von Muskelverletzungen. Rehabilitation und Grenzen}

\begin{abstract}
Zusammenfassung
Häufigkeit, Ursache und Risiken von Muskelverletzungen. Muskelverletzungen im Fußball betreffen in über $90 \%$ der Fälle die untere Extremität und treten in signifikanter Häufung im Wettkampf auf. Ursache für diese Verletzungen ist die Dehnung des Muskels über sein physiologisches Maß hinaus. Als Risikofaktoren sind sowohl das mangelnde Aufwärmen als auch unzureichendes Training oder aber Übertraining zu nennen.

Diagnostik und Therapie. Fast alle akuten Muskelverletzungen sind der konservativen Therapie zugänglich. Es bedarf jedoch der genauen Diagnosestellung und Klassifikation, um die Behandlung stadiengerecht
\end{abstract}

einleiten zu können. Hierzu gehören neben der exakten Anamneseerhebung und ausführlichen körperlichen Untersuchung auch die Sonographie und Magnetresonanztomographie. Neben der supportiven oralmedikamentösen Therapie stehen zudem die komplette Bandbreite der Physiotherapie und ggf. auch die Injektionstherapie zur Verfügung. Nicht zu unterschätzen ist die Prävention, um die Inzidenz von Muskelverletzungen möglichst gering zu halten.

\section{Schlüsselwörter}

Skelettmuskel · Muskelzerrung ·

Muskelfaserriss · Risikofaktoren · Prävention

\section{Conservative treatment of muscle injuries. Rehabilitation and limitations}

\begin{abstract}
Incidence, causes, and risks for muscle injuries. Muscle injuries in soccer appear in over $90 \%$ of cases in the lower extremity and occur more commonly during competition. The reason for these injuries is stretching of the muscle beyond its physiological elasticity. Risk factors include the lack of warming up, inadequate training, or over-training. Diagnosis and therapy. Almost all acute muscle injuries are amenable to conservative therapy. However, precise diagnosis and classification are required prior to starting therapy. Sonography and MRI should also be in-
\end{abstract}

cluded in addition to an exact medical history elevation and detailed physical examination. Besides supportive oral pharmaceutical therapy, the complete range of physiotherapy and, if necessary, injection therapy options are available for treatment. Prevention should not be underestimated to keep the incidence of muscle injuries as low as possible.

Keywords

Muscle, skeletal - Rupture of muscle fiber . Muscle strain · Risk factors · Prevention pie kommen „muscle release“, StrainCounterstrain-Techniken und detonisierende Massagen zum Einsatz. Es folgen der Belastungsaufbau über 1 bis 2 Tage und die Rückführung in die Vollbelastung in Form von nicht ermüdendem Lauftraining und begleitender Physiotherapie.

\section{Typ Ib: Muskelverhärtung durch neurogene Störungen}

Hierunter versteht man neurogene Muskelverhärtungen durch strukturelle oder funktionelle Störungen im Bereich der Lendenwirbelsäule (LWS) und/oder der Iliosakralfugen. Die Patienten beschreiben einen ziehenden/stechenden Schmerz und ein Spannungsgefühl im betroffenen
Muskel. Als typisch in der Anamnese ist die Aussage anzusehen: „Der Muskel hat zugemacht".

Die Therapie beinhaltet die Lösung der neurogenen Störung durch physiotherapeutische und osteopathische Behandlung der betroffenen auslösenden Region der LWS bzw. des Beckens und, je nach Beschwerdebild, ggf. auch die Infiltrationsbehandlung dieser Bereiche und die orale Substitution mit NSAR. Parallel wird mit der Behandlung des betroffenen Muskels, auch hier mit „muscle release“, Strain-Counterstrain-Techniken und detonisierenden Massagen, begonnen. Die Aufbelastung sollte langsam gesteigert werden. Für die ersten 2 Tage empfiehlt 

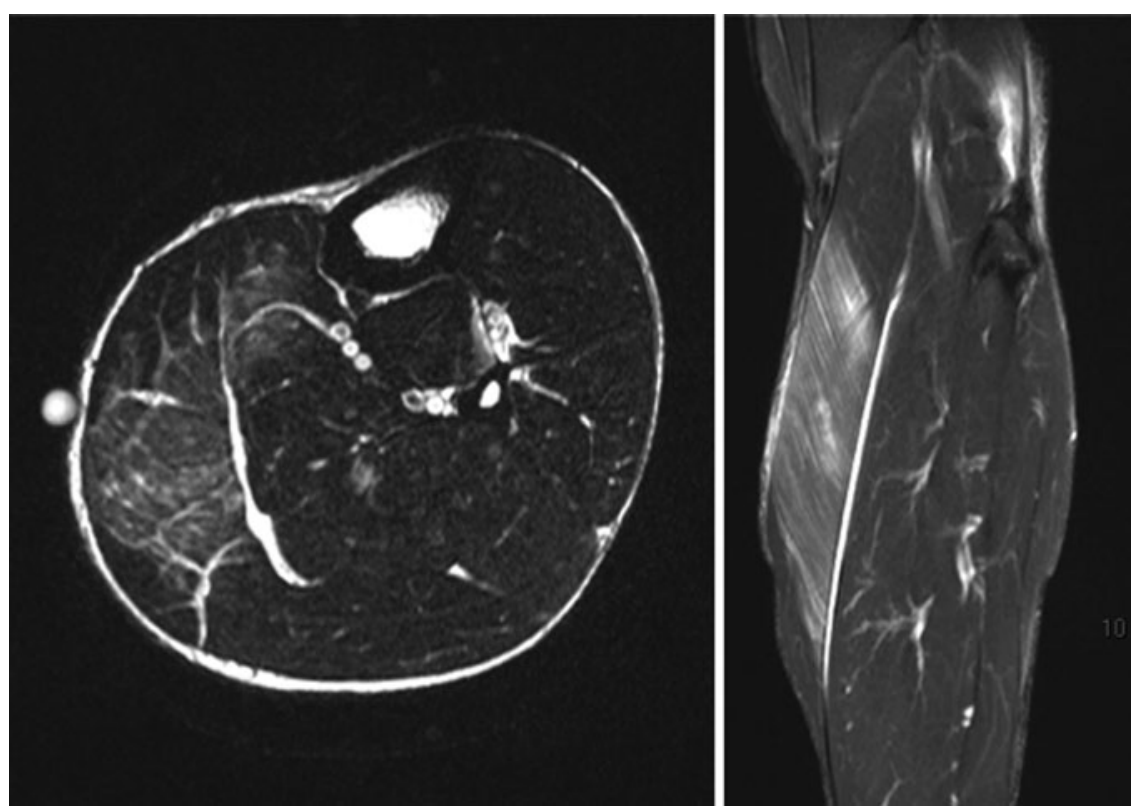

Abb. 1 A Magnetresonanztomogramm einer Typ-II-Verletzung

sich ein ermüdungsfreies Laufen (1. Tag $10 \mathrm{~min}, 2$. Tag $20 \mathrm{~min})$, am 3. Tag die Belastungssteigerung mit 2-maligem Training mit wechselnder Laufgeschwindigkeit und ab dem 4. Tag das Integrationstraining oder aber bei Beschwerdefreiheit auch schon die Wiederaufnahme des Trainings.

\section{Typ II: Muskelzerrung}

Der Unterschied zur Typ-Ib-Verletzung liegt die tatsächliche Schädigung der Muskulatur im mikroskopischen Bereich. Im MRT (Magnetresonanztomogramm) zeigt sich eine flächige ödematöse Veränderung im Bereich des verletzten Muskels (- Abb. 1). Diese kann durch Überlastung und/oder Tonusregulationsstörung entstehen. Die Symptome sind ein plötzliches Ziehen sowie ein zunehmendes Spannungsgefühl, welches häufig in einem krampfartigen Schmerz endet.

Die Therapie entspricht im Prinzip derjenigen bei den Typ-Ib-Läsionen.

\section{Typ Illa: Muskelfaserriss}

Typ-III-Verletzungen sind makroskopisch sichtbar (- Abb.2). Sowohl der Muskelfaser- als auch der Muskelbündelriss entstehen durch Längsdehnung bei aktiver Kontraktion bzw. einem Hypertonus über die Elastizitätsgrenze hinaus. Im Unterschied zu den Typ-I- und -II-Läsionen kommt es zu einem plötzlich auf- tretenden Schmerz, der vom Patienten als stichartiger und spitzer beschrieben wird. Je nach Ausmaß der Verletzung ist eine weitere Belastung nicht möglich.

Bei der Behandlung gilt es, den Patienten nicht $\mathrm{zu}$ früh in die Belastung zurückzuführen und ein abgestuftes Therapiekonzept einzuhalten. Am Verletzungstag sollten Injektionstherapien im verletzten Bereich durchgeführt werden. Des Weiteren wird lokal mittels initial angelegter Kompressionsverbände behandelt, die durch entlastende Salbenverbände abgelöst werden. Zusätzlich sollten dem $\mathrm{Pa}$ tienten muskeldetonisierende Medikamente, NSAR und Supportiva oral zugeführt werden.

Phase 1. Vom 1. bis 3. Tag sollten alle Möglichkeiten der Physiotherapie ausgenutzt werden. Ausnahme bilden Massagen im verletzten Bereich. Um die Wettbewerbsfähigkeit und den Fitnesszustand zu erhalten, sollte ein Training für die gesunden Körperabschnitte mittels Handergometer bzw. Oberkörperkrafttraining durchgeführt werden. Je nach Verletzungsausmaß schließt sich am 2. Tag nach der physikalischen Therapie eine erneute Injektionstherapie an. Ab dem 3. Tag wird im verletzten Bereich mit Strain-Counterstrain-Therapien begonnen.
Phase 2. Vom 4. bis 5. Tag wird im verletzten Areal mittels Elektrotherapie, Ultraschall, Lymphdrainage sowie Massagen ohne direktes Tangieren des verletzten Bereichs behandelt. Gegebenfalls wird am 4. Tag die letzte Injektionstherapie durchgeführt. Ab dem 5. Tag kann vorsichtig mit Querfriktionen begonnen werden. Die verletzte Region wird nun im geschlossenen System angegangen, und es sind isolierte exzentrische Beübung sowie CPM-Maßnahmen („continious passive motion“) erlaubt.

Phase 3. Vom 6. bis 10. Tag liegt der Schwerpunkt in der Trainingstherapie. In der Physiotherapie können nun neben der Elektro- und Lasertherapie die gezielte Massage und Querfriktion angewendet werden. Zusätzlich wird mit ermüdungsfreiem Laufen (etwa $20 \mathrm{~min}$ ) begonnen, was im Verlauf auf 2-mal $20 \mathrm{~min} /$ Tag gesteigert werden kann.

Ab dem 10. bis 12. Tag kann bei subjektiver Beschwerdefreiheit mit Steigerungsläufen bis hin zum Integrationstraining aufbelastet werden.

\section{Typ IIIb: Muskelbündelriss}

Der Muskelbündelriss beinhaltet die Schädigung einer Vielzahl von Muskelfasern, die das Muskelbündel bilden. Meist sind diese Verletzungen aufgrund der entstandenen Lücke gut als Delle tastbar. Die Symptome und die Primärbehandlung entsprechen den Grundzügen derjenigen bei Typ-IIIa-Verletzungen. Allerdings sind die Ausfall- und Behandlungszeit aufgrund des erheblich größeren Verletzungsausmaßes deutlich prolongiert:

Phase 1. In der 1. Woche gilt absolute Sportkarenz.

Phase 2. Ab der 2. Woche wird mit Ultraschalltherapie und erster Bewegungstherapie begonnen, wobei das eigene Körpergewicht nicht überschritten werden sollte. Zur Anwendung kommt auch die propriozeptive neuromuskuläre Faszilitation.

Phase 3. Mit Beginn der 3. Woche kann der Patient mit Aquajogging und Training auf dem Fahrradergometer beginnen. Wichtig sind zudem die neuromuskuläre Bahnung und Massagen proximal und 

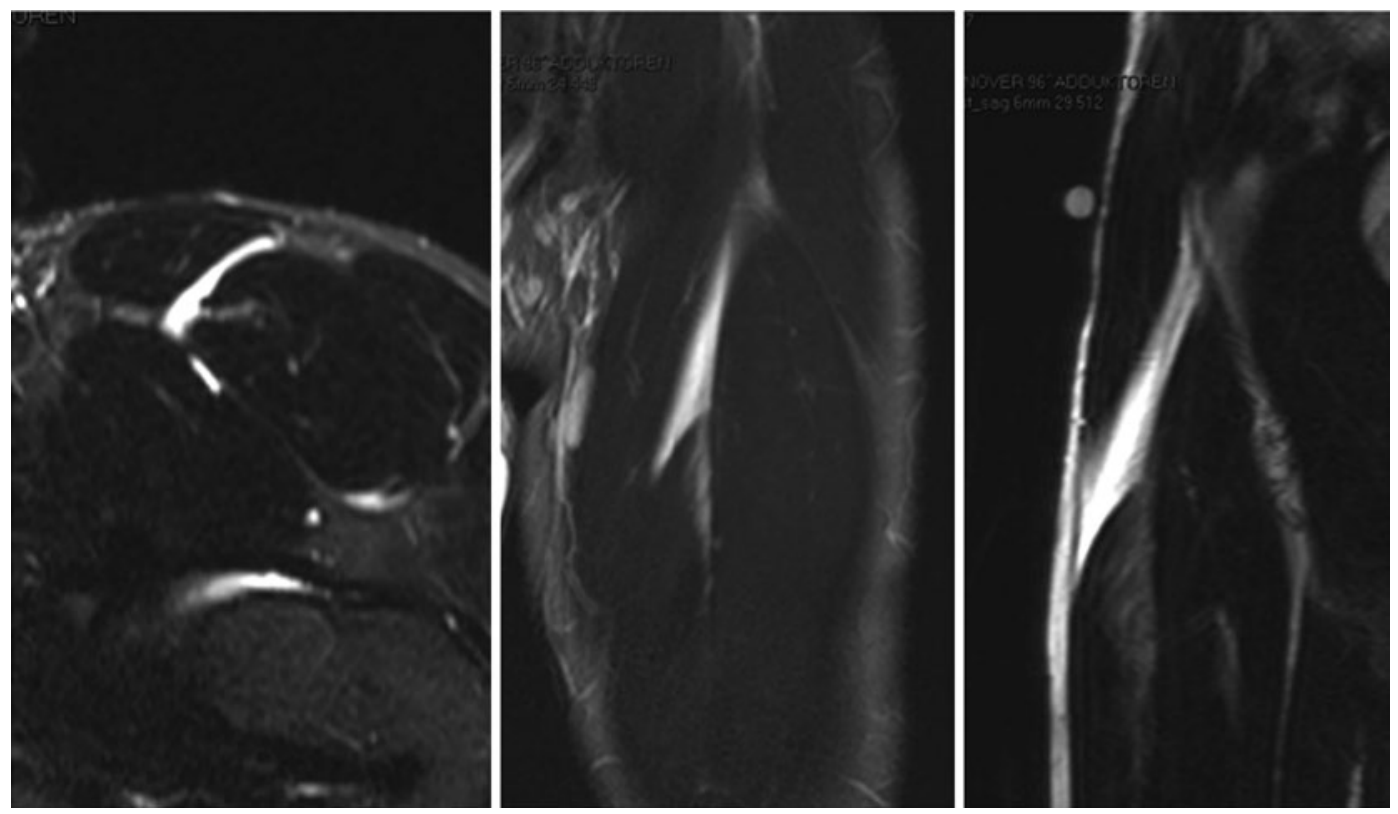

Abb. $2<$ Magnetresonanztomogramm einer Typ-IllaVerletzung
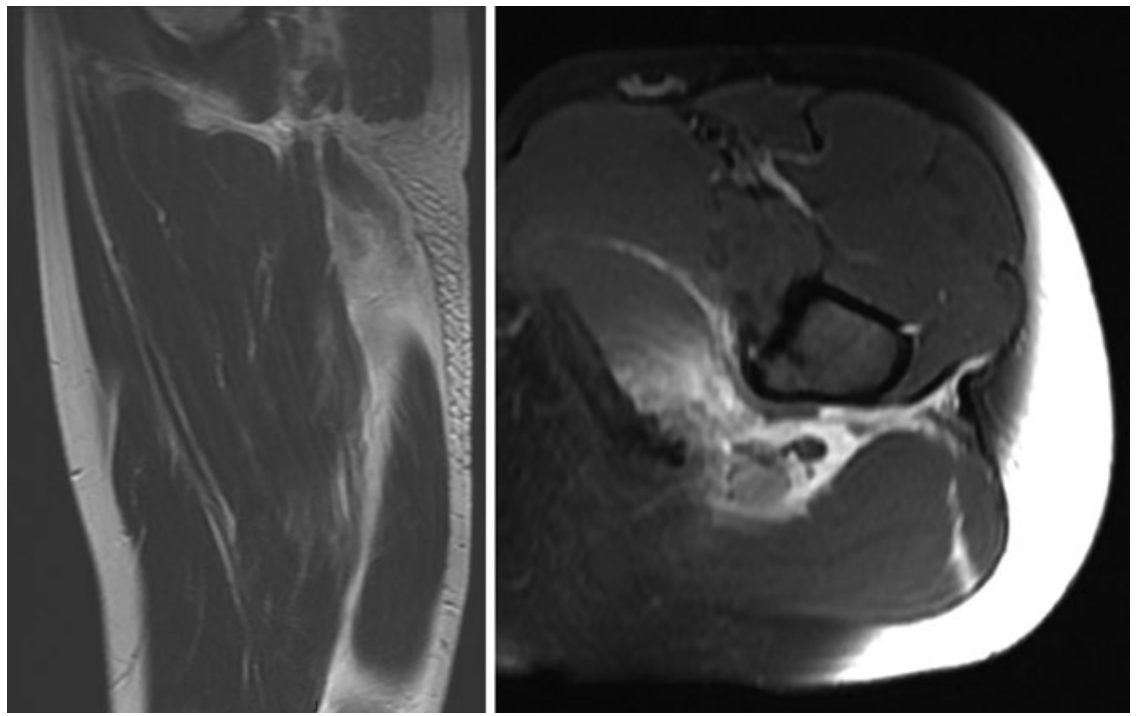

Abb. $3 \Delta$ Magnetresonanztomogramm einer Typ-IIlc-Verletzung

distal der Verletzung sowie die Durchführung von Techniken nach Jander [6].

Phase 4. $\mathrm{Ab}$ der 4 . Woche können die Belastung gesteigert und der Patient über Aquajogging oder Alter-G-Laufband langsam an die Aufbelastung herangeführt werden. Ergänzt wird der Belastungsaufbau durch Gang- und Laufschulung sowie Übungen zur Verbesserung der Propriozeption.

Phase 5. In der 5. Woche wird die Aufbelastung durch Lauftraining mit langsamer Belastungssteigerung mit Fokus auf der Grundlagenausdauer erweitert. Der gesamte Aufbau sollte bis zur 6. Woche langsam gesteigert werden. Aufgrund der Gefahr von heterotopen Ossifikationen sollte die verletzte Region allerdings bis zur vollständigen Resorption des Hämatoms geschont werden.

\section{Typ Illc: Muskelriss}

Direkte Muskelrisse im Bereich des Muskelbauchs sind als Rarität anzusehen und entstehen in der Regel nur durch direkt einwirkende Traumen.

Häufiger sind die Avulsionsverletzungen an den Sehnenansätzen, z. B. dem
Ansatz der ischiokruralen Muskulatur am Tuber ischiadicum (- Abb. 3). Entstehen können sie durch eine Längsdehnung über die Elastizitätsgrenze hinaus. Es resultieren strukturelle Läsionen, welche auch nach nicht ausreichend therapierter Zerrung, Muskelverhärtung oder Muskelfaser-/-bündelriss entstehen können. Im Gegensatz zu den Verletzungen des Muskelbauchs beschreiben die Betroffenen in aller Regel einen schlagähnlichen dumpfen Schmerz. Gerade als frische Verletzung und besonders bei sportlich aktiven Patienten sollten diese Verletzungen der operativen Therapie zugeführt werden. Hierbei können dann alle Möglichkeiten zur Refixation, wie Fadenanker oder auch transossäre Refixationen, zum Einsatz kommen.

\section{Muskelkontusionen}

Sie entstehen immer durch direkte stumpfe Traumen. Der Patient beschreibt einen stumpfen krampfartigen Schmerz und gibt ein Gefühl der Muskelermüdung mit begleitender Belastungsunfähigkeit an. Die Therapie erfolgt analog dem Ausmaß, entsprechend den korrespondierenden Läsionen der indirekten Verletzungen. 


\section{Prävention: Aufwärmen}

Einen wichtigen Teil in der Therapie nimmt indirekt bereits die Prävention von Muskelverletzungen ein.

Soligard et al. [9] konnten in einer Studie mit fast 1900 Spielerinnen aus Norwegen signifikante Verringerungen von Verletzungsrisiko, Überlastungsschäden und schweren Verletzungen nachweisen. Ein wichtiger Teil der Prävention ist neben der gezielten Verbesserung der muskulären Konstitution das Aufwärmen. Es kann zum einen passiv durch Baden, Saunagänge und Stretching erfolgen. Wichtiger jedoch ist die aktive Aufwärmung durch Muskelkontraktionen. Ziel des Aufwärmens ist eine Erhöhung der Körpertemperatur mit konsekutiver Erhöhung der Stoffwechselaktivität. Gleichzeitig sinkt die Muskelviskosität, und sowohl die Ausbreitung als auch die Amplitude des Erregungspotenzials nehmen zu. In der Summe führt dies zu einer gesteigerten Muskelaktivität und einer verringerten Verletzungsanfälligkeit.

Ein gutes Programm zur Prävention von Muskelverletzungen ist das 11+-Programm der FIFA, F-Marc, welches ohne große Umstellung einfach in das Training integriert werden kann [2].

\section{Korrespondenzadresse}

\section{Dr. A. Partenheimer}

Klinik für Unfall- und

Wiederherstellungschirurgie,

Abteilung des BG-Unfallkrankenhauses

Hamburg, Diakoniekrankenhaus

Friederikenstift gGmbH,

Postfach 2040, 30020 Hannover

axel.partenheimer@ddh-gruppe.de

Interessenkonflikt. Der korrespondierende Autor gibt an, dass kein Interessenkonflikt besteht.

The supplement containing this article is not sponsored by industry.

\section{Literatur}

1. Brien S, Lewith G, Walker A et al (2004) Bromelain as a treatment for osteoarthritis: a review of clinical studies. Evid Based Complement Alternat Med 1(3):251-257

2. Deutscher Fussball-Bund (2007) 11+ Ein komplettes Aufwärmprogramm zu Verletzungsprävention. DFB, Frankfurt/Main
3. Ekstrand J, Hägglund M, Walden M (2009) Injury incidence and injury patterns in professional football - the UEFA injury study. Br J Sports Med 45(7):553-558

4. Engelhardt M, Lorbach $O$ (2010) Muskel- und Sehnenverletzungen. Orthopade 39(12):1097

5. Engelhardt M, Krüger-Franke M, Pieper H-G (2005) Sportverletzungen und Sportschäden. Thieme, Stuttgart New York

6. Jander V (2009) Manuelle Muskelfunktionsdiagnostik, 4. Aufl. Elsevier, Urban \&Fischer, München

7. Maurer HR (2001) Bromelain: biochemistry, pharmacology and medical use. Cell Mol Life Sci 58:1234-1245

8. Müller-Wohlfahrt H, Ueblacker P, Hänsel L (2010) Muskelverletzungen im Sport. Thieme, Stuttgart New York

9. Soligard T, Mykleburst G, Steffen K et al (2008) Comprehensive warm up programme to prevent injuries in young female footballers: cluster randomised trial. BMJ 337: a2469

10. Typaldos ST (2010) Orthopadische Medizin: die Verbindung von Orthopäde und Osteopathie durch das Fasziendistorsionsmodell. CIP-Einheitsaufnahme. Die Deutsche Bibliothek, Leipzig

11. Wagner J (2007) Über die Inhaltsstoffe der Roßkastanie. Arzneimittelforschung 2:205-209 